\title{
»Terroristische Netzwerke« und para-totalitäres Strafrechtsdenken
}

Durch das apokalyptische Verbrechen des 11. September 2001 ist das diffuse Gefühl, durch den Islam bedroht zu sein, zu einer - psychologisch gesprochen - generalisierten Angststörung angewachsen, die breite Teile der Bevölkerung erfasst hat. Politik, Medien und Unterhaltungsindustrie spielen auf der Klaviatur der Massenpsychologie und tragen mit ihrem Aktionismus und ihren Dramatisierungen weniger zur Befriedung und Beruhigung als zur weiteren Anstachelung von Ängsten bei. Die Angst vor Terroranschlägen ist berechtigt, wer wollte das bestreiten. Aber ist es tatsächlich gerechtfertigt, von einem qualitativ gänzlich neuen Bedrohungsszenario zu sprechen und als Reaktion mit der Demontage grundlegender Prinzipien und Wertorientierungen des rechtsstaatlich-liberalen Strafrechts zu beginnen?

$\mathrm{Zu}$ dieser Frage möchte ich einige Überlegungen vorstellen. Der begrenzte Rahmen macht es dabei erforderlich, zugespitzt zu argumentieren. Manche Schärfe der Betrachtung ist dabei aber auch deswegen unvermeidlich, weil diejenigen, die für eine Neuorientierung des Terrorismusstrafrechts als Feindstrafrecht eintreten, laute kriegerische Töne anschlagen. Lassen sie mich zunächst die Phänomene beschreiben, um die es hier geht:

Auffällig ist bereits der Umstand, dass im Zusammenhang mit den Taten islamistischer Terroristen zwar einerseits Organisationsnamen wie die Al Kaida genannt werden, zugleich aber immer wieder betont wird, dass es sich nicht um Organisationen im überkommenen Sinne, sondern um »Terrornetzwerke« handle. Dieser diffuse Begriff hat zur Folge, dass übliche Kategorien, wie wir sie aus der Dogmatik der Täterschaft und Teilnahme und aus den Organisationsdelikten kennen, nicht mehr präzise zu greifen vermögen. Mitgliedschaft oder Sympathisantentum zu Al Kaida kann sich nach der juristischen und/oder geheimdienstlichen Betrachtung - wobei beides immer mehr miteinander verschwimmt - etwa aus dem Umstand ergeben, dass sich eine Person zu einer bestimmten Zeit in einem Taliban-Ausbildungslager in Afghanistan befunden oder eine bestimmte Koranschule besucht haben soll, Kontakt zu bestimmten islamischen Predigern gehabt haben soll, zu den regelmäßigen Besuchern einer bestimmten Moschee zählt oder für bestimmte islamische Wohlfahrtsverbände gespendet oder gesammelt hat. Es gibt also keine Mitgliedschaften in einer Vereinigung mehr, sondern Personen sind auf die eine oder andere Weise miteinander und mit dem Terror verknüpft. Dabei können diese Verknüpfungen auch aus allgemein üblichen und verbreiteten religiösen und kulturellen Gebräuchen bestehen - wie etwa dem Brauch, dass die gemeinsame Pilgerfahrt nach Mekka Menschen zu unbedingter Solidarität in der Bewältigung von Schwierigkeiten des Alltags wie der Erledigung von Geldgeschäften, Wohnungsanmietungen usw. verpflichtet.

Auch die Kategorie der psychischen Beihilfe verschwimmt, wenn sie mit dem Bild des terroristischen Netzwerkes verknüpft wird: Soll etwa jedes Mitglied eines Freundes- und Glaubensbrüderkreises, der sich verbal radikalisiert, als Gehilfe derjenigen Täter eingestuft werden, die den vielleicht nur kurzen Schritt von der Propaganda des Wortes zur Propaganda der Tat gehen? Verwandelt sich die Nicht-Denunziation einer derartigen erkennbaren Gewaltbereitschaft in strafbare Beihilfe? 
Die begriffliche Systematik des Strafrechts wird noch durch weitere Phänomene und ihre Verarbeitung durch Geheimdienste, Politik und Medien durcheinander gebracht. So soll es in Europa Hunderte von sogenannten Schläfern geben, die jederzeit - von wem auch immer - geweckt werden können, und sich dann von unauffälligen Migranten in mörderische Kulturkämpfer verwandeln. Wie erkennt man derartige Schläfer empirisch und wie ist dieser Zustand normativ zu bewerten? Als Verbrechensverabredung zu unbestimmten Taten zu einem ungewissen Zeitpunkt, als abstrakt gefährliches Verhalten, als Organisationsdelikt?

Bereits an dieser kurzen Skizze ist zu erkennen, dass übliche Kategorien der individuellen Täterschaft, von Täterschaft und Teilnahme, von Mitgliedschaft in einer terroristischen Vereinigung, von strafloser Vorbereitung und strafbarem Versuch nur schwer mit den polizeilich-geheimdienstlich geschaffenen und von der Politik übernommenen Bildern des modernen islamistischen Terrorismus kompatibel sind. Das Bild der Netzwerke löst soziologisch betrachtet die Kategorie des Akteurs und juristisch betrachtet die dogmatische Figur der individuellen Täterschaft tendenziell auf. Islamistischer Terror breitet sich gleichsam wie ein Rhizom unter der Oberfläche der Gesellschaft aus und schießt dann völlig unberechenbar ohne erkennbare hierarchische Anleitungs- und Befehlsstrukturen irgendwo aus dem Boden. Für eine militärische Strategie des Umgangs mit einem derartigen Bedrohungsszenario ist die Vorgehensweise der US-Armee gegen die Trampelpfade des Vietkong im vietnamesischen Dschungelkrieg sinnfällig: Man kippte flächendeckend über 50 Millionen Liter des hoch giftigen Entlaubungsmittels Agent Orange auf die vermuteten logistischen Strukturen des Feindes ab.

Im Strafrechtsdenken und in der Strafrechtspraxis in Auseinandersetzung mit dem islamistischen Terrorismus sind leider vergleichbare Tendenzen erkennbar, die unsere gewachsene liberal-rechtsstaatliche Strafrechtskultur zu vergiften drohen. Ich möchte hierzu zunächst Tendenzen der internationalen Strafrechtspraxis oder besser: Staatspraxis in der Auseinandersetzung mit dem islamistischen Terror skizzieren.

Die deutlichste und radikalste Tendenz ist die völlige Abkehr vom Strafrecht, ja selbst vom Kriegsrecht hin zu einer rein militärischen Lösung des Problems. Hierfür steht das Konzept des Enemy Combatant, ${ }^{1}$ das nicht nur mit dem Internierungslager von Guantánamo einhergeht, sondern darüber hinaus von Folter, extralegalen Entführungen und Gefangenentransporten und schließlich sogar extralegalen Exekutionen begleitet wird. Die Auseinandersetzung mit dem Terrorismus heißt in diesem Konzept »war against terrorism «, ${ }^{2}$ wird vom Militär, den Geheimdiensten und privaten Sicherheitsunternehmen im Auftrag der Regierung geführt. Eine Art Ausnahmezustand wird

1 die »Rechts«grundlage für die Festsetzung und Behandlung feindlicher Kämpfer bildet die Military Order des Präsidenten George W. Bush vom 13.11.2001 Detention, Treatment, and Trial of Certain Non-Citizens in the War Against Terrorism, vollständiger Text unter http:// www.whitehouse.gov/news/releases/2001/11/print/20011113-27.html

2 der Begriff des »war on terrorism « wurde von George W. Bush anlässlich seiner Rede vom 20.9.2001 vor dem Senat und Kongress in den politischen Diskurs eingeführt, der vollständige Text der Rede findet sich unter http://www.whitehouse.gov/news/releases/2001/09/ 20010920-8.html 
behauptet, die Justiz wird an den Rand des Geschehens gedrängt, Menschenrechte und Verfassungsgarantien werden suspendiert. ${ }^{3}$ Von der Behandlung, die Menschen widerfährt, die einem solchen Regime unterworfen sind, kann man sich durch Interviews mit ehemaligen Guantánamo-Gefangenen ein Bild machen. ${ }^{4}$ Unter noch ganz anderen Umständen wird seit drei Jahren Ramzi Binalshibh gefangen gehalten. Er befindet sich an einem unbekannten Ort auf dieser Welt, höchstwahrscheinlich außerhalb der USA, ohne jeden rechtlichen Beistand und seine Aussagen kursieren als sogenannte unclassified summaries geheimdienstlicher Vernehmungen überall auf der Welt durch Al Kaida-Strafverfahren, ohne dass jemals einer der Verfahrensbeteiligten Binalshibh zu Augen bekommen würde. ${ }^{5}$ Dass unter solchen Bedingungen als Strategie des »war against terrorism « auch die Manipulation justizieller Verfahren durch Beweisselektion und Zeugenentziehung befürchtet werden muss, hat der 3. Strafsenat des BGH unter richterlicher Zurückhaltung, aber doch mit der gebotenen Deutlichkeit festgestellt. ${ }^{6}$

Die Legitimation für dieses Konzept ist schnittig und einfach: Bei den islamistischen Terroristen handelt es sich um Täter, die auch Massenmorde an Zivilisten planen und in die Tat umsetzen, und dabei keinerlei Achtung für Menschenrechte und ihre Mitmenschen zeigen. Derartige feindliche Kämpfer verdienen nicht die Einhaltung humanitärer Standards - so lässt sich das plakativ und erfolgreich an große Teile der Öffentlichkeit verkaufen.

Subtiler - und dadurch für das liberal-rechtsstaatliche Strafrecht fast noch gefährlicher - ist eine Denkensart, in der Strafrecht, Kriegsrecht und Notstandsrecht miteinander verschmolzen werden, um daraus eine moderne, als angemessen hingestellte Waffe gegen den Terrorismus neuer Art zu schmieden.

Schon in der deutschen Diskussion der 70-er Jahre des vergangenen Jahrhunderts wurde die Diskussion um neue erforderliche Bekämpfungs- und Sicherheitsgesetze von kriegerischen und apokalyptischen Szenarien begleitet: die RAF habe dem Staat den Krieg erklärt, es sei mit Angriffen auf die Trinkwasserversorgung von Großstädten und auf Fußballstadien zu rechnen usw. usf. Im Zusammenhang mit den Ereignissen des Herbst 1977 kamen Diskussionen um die Relativität des Folterverbotes und zur Notwendigkeit von erweiterten Notstandbefugnissen auf. Niemand stellte jedoch in Abrede, dass es sich bei terroristischen Akten um Straftaten handelt, deren Beurteilung in die Zuständigkeit ordentlicher Gericht fällt und die durch das übliche Sankti-

3 nach der Analyse von Richard Jackson, Writing the War on Terrorism, Language, politics and counter-terrorism, Manchester/New York 2005 lauten die zentralen Botschaften dieses Konzepts wie folgt: die Anschläge vom 11. September 2001 seien ein kriegerischer Akt gewesen; die Terroristen seien inhumane Barbaren, die es verdient hätten, von der zivilisierten Gesellschaft ausgerottet zu werden; die Bedrohung durch den Terrorismus sei katastrophal und es sei nur rational, hierauf mit allen Streitkräften zu antworten; der USamerikanisch geführte »Krieg gegen den Terrorismus « sei per definitionem ein guter und gerechter Krieg. Vgl. dazu auch American Civil Liberties Union (ACLU), Civil Liberties after 9/11, unter: http://www.aclu.org/FilesPDFs/911_report.pdf

4 Roger Willemsen, Hier spricht Guantánamo, Frankfurt a.M. 2006

5 vgl. dazu das Feature des Deutschlandfunks vom 11.4.2006: Feindstrafrecht? Über Terroristenbekämpfung im Rechtsstaat, Manuskript abrufbar unter: http://www.dradio.de/down$\operatorname{load} / 51125$

6 BGH 3 StR 269/04 = NJW 2005, 2322 ff. 
onsinstrumentarium zu ahnden sind. Die Ereignisse des 11. September haben zu einem dramatischen Verfall dieser traditionellen Sichtweisen geführt. Aber welche Berechtigung sollte es dafür geben, dass mit sogenannten Ticking-bomb-Beispielen ${ }^{7}$ das Folterverbot durchlöchert wird, eine Einschränkung von Verfahrensgarantien für Terroristen für möglich und eine sicherungsverwahrungsähnliche Unterbringung für erforderlich gehalten wird? Ein signifikanter Unterschied der Taten von New York, Madrid und London gegenüber früheren Terrorakten kann in dem quantitativen Sprung der Menschenopfer und der Zerstörungskraft und in der besonderen Qualität von Selbstmordattentaten gesehen werden. Die quantitative Dimension stellt für das traditionelle Strafrechtsdenken indes keine besondere Schwierigkeit der Bewältigung dar. Es ist für das Strafrechtsdenken eine übliche Differenzierung, dass sich nach dem Parameter Schadenquantität einfache und besonders schwere Fälle eines Tatbestandes unterscheiden lassen, dass sich an dem Grad des Unrechts die Zuständigkeit des Gerichts orientiert, und dass die Folgen der Tat Relevanz für die Strafzumessung besitzen. Es ist dem Strafrechtssystem sogar gelungen, Massenmorde und Regierungskriminalität noch viel größeren Ausmaßes als jeder Terrorakt bisher rechtsstaatlich aufzuklären und zu verarbeiten. Es muss also einen anderen Grund dafür geben, warum man meint, gegen den Terrorismus eine Art Recht schmieden zu müssen, das weniger mit Strafgerechtigkeit als mit grober staatlicher Gewalt zu tun hat. Der entscheidende qualitative Aspekt scheint mir die Struktur des Selbstmordattentates zu sein. Die unbedingte Entschlossenheit zur Tat, Selbstmordattentäter, die den Tod wünschen, die nicht mehr zur Verantwortung gezogen werden können, und die der weltlichen Macht auch jedes Recht abstreiten würden, ihre Taten zu verdammen, lösen offenbar ein unerträgliches Gefühl von Wehrlosigkeit und in der Reaktion einen unbedingten Willen aus, sich dagegen behaupten zu können. Nur in dieser psychologischen Dimension lässt sich verstehen, wie völlig irrationale Strategien wie Folterung und sicherndes Wegsperren plötzlich als erforderliche und erfolgreiche Mittel zur Bekämpfung des Terrorismus verkauft werden. Es ist frappierend, wie die luziferische Konstruktion des islamistischen Terrors in den Medien und der Politik von der Wiederkehr inquisitorischer Verfolgungs- und Überführungsstrategien begleitet wird. Wenn es nur gelänge, die bösen Männer mit Bärten aus ihren afghanischen Höhlen zu entführen, ihnen ihre Verbindungen abzufoltern und sie dann auf ewig irgendwo in Verliesen verschwinden zu lassen, dann soll offenbar das Problem des islamistischen Terrorismus seiner Lösung ein Stück näher gekommen sein.

Das Gegenteil hiervon dürfte richtig sein: Dem Terrorismus werden Rekrutierungschancen und ideologische Munition verschafft, wenn die Bekämpfung des Terrorismus im Stil von Abu Ghraib, Guantanamo und Militärtribunalen durchgeführt wird. Derartige Ansätze eines Sonderrechts, einer Sondergerichtsbarkeit und einer Spezialbehandlung des Terrorismus tragen die Gefahr in sich, dass die moralische Überlegenheit freiheitlich demokratischer Gesellschaften und ihrer rechtlichen Verfassung als Rechtsstaat, dass ihre Legitimation aus dem Respekt vor den Menschen- und Bürger-

7 vgl. dazu Heiner Bielefeldt, Das Folterverbot im Rechtsstaat, Deutsches Institut für Menschenrechte (Policy Papers No. 4), Berlin 2004, S. 7 f. m.w.N. 
rechten erheblichen Schaden nehmen. Eine solche Vorgehensweise führt über die direkten Rekrutierungswirkungen hinaus zur Solidarisierung ganzer Landstriche, Kulturen und Religionsgemeinschaften mit den Betroffenen. Am sogenannten Karikaturenstreit der letzten Monate hat man gesehen, welche Problemverschärfungen dies zur Folge haben kann.

Es ist nicht bestreitbar, dass Wege gefunden werden müssen, um unsere Gesellschaft erfolgreich gegen den Terrorismus zu schützen. Zu diesen Wegen gehört aber als paradoxe Vernunft auch, zum Schutz der Terroristen als Rechtspersonen zu stehen. Unter allen Umständen muss ihnen gegenüber das Verbot der Folter, einer inhumanen oder demütigenden Behandlung aufrecht erhalten werden, sie müssen unter allen Umständen das Recht auf einen fairen Prozess behalten und dürfen bei der Strafvollstreckung nicht auf ewig in einer Art von Sicherungsverwahrung ohne Rechte und Aussichten auf Freiheit verschwinden.

Diese klassischen rechtsstaatlichen Positionen werden freilich in der dritten Tendenz - mit der ich abschließen möchte - auf intellektuell elegante, in der Sache aber nicht weniger gefährliche Art und Weise hinwegdisputiert. In der Tradition von Carl Schmitt, der bereits 1932 die »Friedloslegung, hors-la-loi-Setzung und innerstaatliche Feinderklärung ${ }^{8}$ zum Inbegriff des Politischen und damit eines handlungsfähigen Staates erklärt hatte, tritt heute Günter Jakobs immer radikaler dafür ein, sich zu der realen Entwicklung eines von ihm so genannten Feindstrafrechts affirmativ zu verhalten: »Der prinzipiell Abweichende....kann nicht als Bürger behandelt, sondern muss als Feind bekriegt werden «, ${ }^{9}$ heißt es bei Jakobs. Und weiter, nach der rhetorischen Frage, ob mit den Mitteln eines rechtsstaatlichen Strafrechts der Krieg gegen den Terror geführt werden kann: »Ein alles umfassender Rechtsstaat könnte diesen Krieg nicht führen; denn er müsste seine Feinde als Personen und dürfte sie demgemäß nicht als Gefahrenquellen behandeln. Beim praktisch optimalen Rechtsstaat verhält es sich anders, und das bringt ihm die Chance, nicht an den Attacken seiner Feinde zu zerbrechen «. ${ }^{10}$

Der »praktisch optimale Rechtsstaat« ist ein Euphemismus für ein para-totalitäres Strafrecht, das nach der Theorie von Jakobs jeden, der nicht »einigermaßen verlässlich Rechtstreue leistet,..., nicht als Person behandeln $\aleph^{11}$ und das die Bekämpfung des Ter-

8 C. Schmitt, Der Begriff des Politischen, Berlin 1932; in dieser Schrift entwickelt Schmitt jenen Begriff aus dem Kampf zwischen »Freund« und »Feind« Die Begriffe »Freund«, »Feind « und »Kampf « sind dabei in ihrer realen sinnlich-praktischen Gestalt gemeint, so dass der »Kampf « nicht etwa als Metapher für geistiges Ringen steht, sondern von Blut durchströmt wird. Er beinhaltet damit die Möglichkeit der physischen Tötung - über den, der friedlos und aus dem Recht gestellt ist, wird notfalls auf der Stelle gerichtet (wie man dann an der Rechtfertigung des blutigen Vorgehens gegen den sog. Röhm-Putsch durch Carl Schmitt, Der Führer schützt das Recht, in: DJZ 1934, Sp. 945 ff, sehen kann, dort findet sich auch der bezeichnenden Satz: »Für die Rechtsblindheit des liberalen Gesetzesdenkens war es kennzeichnend, daß man aus dem Strafrecht den großen Freibrief, die ,Magna Charta des Verbrechers' (Fr. von Liszt) zu machen suchte«.

9 Jakobs, Bürgerstrafrecht und Feindstrafrecht, HRR-Strafrecht (Onlinezeitschrift) 3/2004, 88 ff. (95), abrufbar unter: http://www.hrr-strafrecht.de/hrr/archiv/04-03/hrrs-3-04.pdf

10 Jakobs, Terroristen als Personen im Recht?, ZStW 117 (2005), 839 ff. (851)

11 a.a.O., 843 
rors als »ein Unternehmen gegen Feinde $\ll^{12}$ betreiben müsste, das sich bei »Maßnahmen zur Vermeidung des extremen Notfalls, die sich gegen Terroristen, also gegen die Urheber des Notfalls, richten,.......keine Tabus auferlegen ${ }^{13}{ }^{13}$ dürfte, ja, Terroristen vor allem deswegen »nicht mehr als Personen behandeln darf, weil er ansonsten das Recht auf Sicherheit der anderen Personen verletzen würde «. ${ }^{14}$

Auch wenn Jakobs für sein Konzept des Feindstrafrechts rechtsphilosophische Kronzeugen von Rousseau über Fichte bis Kant auffährt ${ }^{15}$ und scharfsinnig argumentiert, lässt sich nicht verbergen und wird von Jakobs auch nicht verborgen, dass sein Text von der petitio principii ausgeht, dass wir uns heute in einer Art Weltkrieg gegen den Terrorismus befinden. Diese petitio principii verstrickt sich auf fatale Weise im Terrorismus. Hinter den realisierten und geplanten apokalyptischen Anschlägen fanatischer Terroristen ist genau die Absicht zu vermuten, dass die freiheitlich demokratischen Staaten ihre moralische Überlegenheit kraft ihrer Legitimation aus dem Respekt vor den Menschen- und Bürgerrechten panikartig hinter sich lassen und so in ihrem Krieg gegen den Terrorismus selbst zu dessen Krieg gegen die Zivilisation beitragen.

Rechtskultur muss sich aber in einer solchen Situation von Hysterie und Übermaß fernhalten, sie darf sich nicht auf den Leim der Entwicklung überlegener (militärischer) Bekämpfungsstrategien locken lassen und sie muss sich bemühen, aus der Erhabenheit von Rechtsprinzipien Gelassenheit zu schöpfen. So ist etwa den Hütern und Hüterinnen der deutschen Verfassung in Karlsruhe großer Respekt und Dankbarkeit dafür zu erweisen, dass sie mit ihrem Urteil von 15. Februar $2006^{16}$ das Luftsicherheitsgesetz, das den Abschuss einer von Terroristen entführten Passagiermaschine samt Passagieren legitimierte, für verfassungswidrig erklärt haben und damit der Militarisierung der Bekämpfung des Terrorismus die absolute Schranke der Menschenwürdegarantie in den Weg gestellt haben. ${ }^{17}$ Ebenso sollte man allen Konzepten eines paratotalitären Strafrechts die Menschenwürde und die aus ihr ableitbaren Garantien des Respekts gegenüber jeder Person und der für jede Person gebotenen fairen Behandlung entgegenstellen. Unsere Gesellschaft droht nicht an mangelnder Entschlossenheit gegenüber dem Terrorismus zugrunde zu gehen, sondern daran zu verderben, dass sie ihre freiheitlichen Ideale und Prinzipien in der Auseinandersetzung mit dem Terrorismus aushöhlt und sogar gänzlich aufgibt.

12 a.a.O., 846

13 a.a.O., 848

14 Jakobs, Bürgerstrafrecht und Feindstrafrecht 3/2004, 88 ff. (93)

15 a.a.O., $89 \mathrm{ff}$.

161 BvR 357/05 = NJW 2006, $751 \mathrm{ff}$.

17 vgl. dazu demnächst ausführlich Herzog, Die Menschenwürde als absolute Grenze instrumenteller Vernunft, Zur Frage von Abwägungsverboten aus Anlass des Luftsicherheitsgesetzes (erscheint September 2006 in der Festschrift für Burkhard Hirsch) 